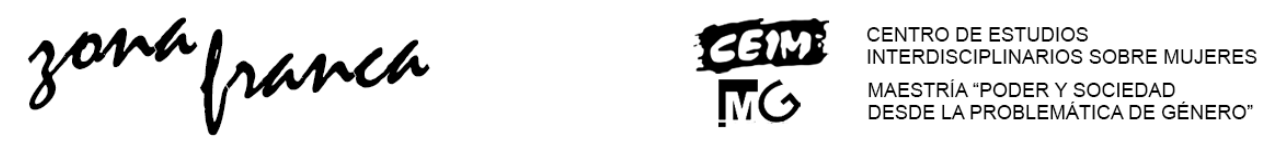

\title{
El género en el estudio de sistemas electorales. Análisis de la ciencia política argentina
}

\author{
María Emilia Perri
}

\section{Resumen}

El estudio de los sistemas electorales ocupa un lugar central dentro de la ciencia política argentina. A partir de ellos, comenzó una nueva línea de investigación que buscaba transparentar las diferencias de género en política y el rol de los sistemas electorales.

Las producciones al respecto demuestran un claro lineamiento con las posturas institucionalistas, donde lo importante es analizar cómo afectan las reglas, normas o conductas sociales a los diferentes actores políticos. Ello nos permite realizar un racconto de lo sucedido dentro de la disciplina argentina y pensar los caminos futuros.

En este trabajo, se analizará las formas en que las categorías de género han sido incorporadas en las investigaciones respecto de los sistemas electorales y de la participación en la ciencia política argentina. ¿Qué ha quedado por fuera de estos estudios? ¿Qué otras líneas de investigación se proponen?

Palabras Claves: Participación política - Ciencia política - GéneroSistemas electorales

\section{Gender in electoral system studies. Analyses of Argentinean political sciences}

\section{Abstract}

* Licenciada en ciencia política por la Universidad Nacional del Litoral. Becaria doctoral, Instituto de Humanidades y Ciencias Sociales, Consejo Nacional de Investigaciones Científicas y Técnicas. Contacto: maemilia.perri@gmail.com

Perri, María Emilia. "El género en el estudio de sistemas electorales. Análisis de la ciencia política argentina" en Zona Franca. Revista del Centro de estudios Interdisciplinario sobre las Mujeres, y de la Maestría poder y sociedad desde la problemática de Género, №27, 2019 pp.197-217. ISSN, 2545-6504 Recibido: 28 de junio 2018; Aceptado: 10 de octubre 2019

Revista Zona Franca- Centro de estudios interdisciplinario sobre las mujeres (CEIM)- Maestría poder y sociedad desde la problemática de género (MG), Rosario, Argentina. ISSN, 2545-6504 http://zonafranca.unr.edu.ar/index.php/ZonaFranca| Numero 27 (2019). 
The study of electoral systems occupies a central place in Argentine political science. From them, began a new line of research that sought to make transparent gender differences in politics and the role of electoral systems.

The productions in this matter show a clear alignment with the institutionalist positions, where the object is to analyze how the rules, norms or social behavior affect the different political actors. This allows us to make a summary of what happened within the Argentine discipline and think about future paths.

In this paper, we will analyze the ways in which gender categories have been incorporated in research regarding electoral systems and participation in Argentine political science. What has been left out of these studies? What other lines of research are proposed?

Key Words: Political participation - Political science- GenderElectoral systems.

\section{Introducción}

Dentro de la ciencia política argentina, ocupa un lugar central el estudio de los sistemas electorales y las maneras en que ello afecta el sistema político y a los individuos que en él se encuentran (Duverger 1955; Sartori 2007,2009). En este sentido, en las últimas décadas el análisis de las diferencias de género en estos procesos se ha transformado en una robusta línea de investigación (Dahlerup 2002; Krook 2009; Norris 1985).

No obstante, en dichos trabajos se observa un enclave institucionalista muy fuerte que se traduce en trabajos respecto de la representación descriptiva de varones y mujeres (Archenti y Tula 2014a; Llanos y Sample 2008; Marx, Borner y Caminotti 2006). Este fuerte mainstream institucionalista se acopla al desarrollo de la ciencia política argentina, que recientemente comienza a buscar nuevas miradas respecto de los fenómenos políticos y electorales.

Dicha perspectiva surge desde Estados Unidos y se reproduce en todos los cónclaves académicos hasta el día de la fecha. Esta línea de análisis se ha enfocado en la manera en que las instituciones afectan el desarrollo de un sistema político y de los individuos que lo componen. La impronta que la misma ha tenido en el desarrollo de la ciencia política argentina es innegable cuando vemos la

Revista Zona Franca- Centro de estudios interdisciplinario sobre las mujeres (CEIM)- Maestría poder y sociedad desde la problemática de género (MG), Rosario, Argentina. ISSN, 2545-6504 http://zonafranca.unr.edu.ar/index.php/ZonaFranca| Numero 27 (2019). 
manera de abordar el estudio de los sistemas electorales con perspectiva de género.

Estos últimos llevan más de veinte años de producción científica argentina. Con lo cual es posible realizar un racconto de lo sucedido y pensar los caminos futuros en el contexto disciplinar.

Por lo antes mencionado en el presente trabajo se buscará observar la manera en que el género ha sido incorporado como variable de análisis en las investigaciones sobre sistemas electorales y participación política; principalmente en la academia argentina.

Para ello, primero se establecerá cuál ha sido esta corriente principal dentro de la ciencia política argentina, y cómo ello ha impactado en el área de conocimiento que aquí nos interesa. Luego se analizará específicamente los trabajos respecto de los sistemas electorales, la participación política y el género en Argentina y se realizarán algunas reflexiones en torno de lo que resta alcanzar.

Finalmente, las preguntas que se intentarán responder serán si existen vías alternativas al institucionalismo dentro de la ciencia política argentina. ¿Qué ha quedado por fuera de dicho análisis? ¿Qué otras líneas de investigación se proponen al margen del institucionalismo?

\section{El mainstream: ¿qué es el institucionalismo?}

El nuevo institucionalismo como paradigma dentro de la ciencia política surge a partir de 1970 y partirá de la oposición a la cosmología comportamentalista: el comportamiento humano no es libre de restricciones constitucionales y el mundo político es un mundo institucional. March y Olsen (1993) establecen que el nuevo paradigma institucionalista parte de la noción de que las instituciones políticas tienen una relativa autonomía, entendiendo que el Estado no sólo se ve afectado por la sociedad, sino que también la afecta.

Revista Zona Franca- Centro de estudios interdisciplinario sobre las mujeres (CEIM)- Maestría poder y sociedad desde la problemática de género (MG), Rosario, Argentina. ISSN, 2545-6504 http://zonafranca.unr.edu.ar/index.php/ZonaFranca| Numero 27 (2019). 
El paradigma piensa y analiza el diseño de las instituciones políticas, y para esto recupera del Antiguo Institucionalismo la concepción de que las instituciones deben ser entendidas como variable explicativa, mientras que mantiene del comportamentalismo el rigor metodológico y la orientación cuantitativa y empírica. Por lo tanto, transforma la cosmología pero mantiene la metodología del paradigma anterior.

Es una vertiente analítica que observa todas las reglas de interacción política (incluidas las económicas y las sociales) y así amplía la concepción de lo que debe entenderse por instituciones. En este contexto los actores políticos deben reflejar los valores de las instituciones con las que se asocian, las cuales se asume que influencian a los actores (Peters 1999).

Mackay, Kenny y Chapell (2010) establecen que el Nuevo Institucionalismo permite comprender las maneras en que las instituciones modelan el comportamiento de los individuos mediante reglas, normas y políticas.

En el mismo período, las teorías feministas construyeron diferentes líneas teóricas buscando transparentar las maneras en que la mujer se constituyó a lo largo de la historia en sujeto oprimido. Dentro de estos trabajos encontramos el rol que el Estado ha tenido en ese desarrollo. Autoras como Pateman (1996) o Phillips (1996) nos ayudan a pensar las diferencias, en cuanto a inclusión y ciudadanía, que presentaron a lo largo de la historia mujeres y varones.

En los '60 y '70 los estudios anglosajones estuvieron signados por la corriente de "Estudio de la Mujer", la cual posiciona el concepto mujer como una categoría de análisis dentro de las ciencias sociales. Pero el principal problema aquí, radica en que esa categoría hace referencia a la mujer blanca y burguesa; hay una ausencia del tratamiento de las intersecciones entre clase, raza y sexo. A raíz de ello, a partir de los 80, surgen los Estudios de Género para superar dichas limitaciones.

Revista Zona Franca- Centro de estudios interdisciplinario sobre las mujeres (CEIM)- Maestría poder y sociedad desde la problemática de género (MG), Rosario, Argentina. ISSN, 2545-6504 http://zonafranca.unr.edu.ar/index.php/ZonaFranca| Numero 27 (2019). 
Ello tuvo su correlato dentro del institucionalismo, en donde surgió una manera de analizar las instituciones desde los géneros, particularmente desde el feminismo: El Institucionalismo Feminista (a partir de aquí IF). Éste vincula dos líneas de análisis muy diferentes: pone a dialogar los aportes del neoinstitucionalismo con las teorías feministas. De esta manera, se busca indagar acerca de la manera en que las instituciones afectan al desenvolvimiento de grupos minoritarios, como el colectivo femenino.

EI IF intenta explicar las diferencias de género entre varones y mujeres, suponiendo que las instituciones también tienen injerencia en la ampliación o disminución de las brechas de género. Desde esta mirada, las instituciones formales e informales y cómo ellas operan, importan. Pero ¿cómo interviene el género dentro de ellas? La principal respuesta es que el género se encuentra en las dinámicas centrales de las instituciones. Krook y Mackay (2011) establecen que argumentar que una institución se encuentra generizada significa que lo masculino y lo femenino están entrelazados en la cultura diaria de las instituciones. El vínculo entre institucionalismo y feminismo puede considerarse como una relación de mutuo beneficio (Mackay, Kenny y Chapell 2010).

Ahora bien, ¿por qué utilizar este encuadre teórico para observar la participación de mujeres en política? Existen instituciones políticas que pueden afectar la forma en que se incluyen las mujeres en el contexto político; instituciones como los partidos políticos, los sistemas electorales o la legislatura, y también instituciones no formales como normas de comportamiento implícitas que se establecen hacia dentro de las anteriores o pautas sociales y culturales que las atraviesen.

Estas instituciones modifican la participación de los diferentes actores en política, establecen determinados procesos de selección de candidatos y particulares maneras de construir las carreras políticas. En este sentido, el IF pone 
el enfoque en el contexto institucional, entendiendo que es éste el que determina el impacto de las reformas electorales (Bluckley, Mariani y White 2014).

En esta línea de análisis, no sólo pensamos en el sistema electoral como una regla formal que determina las posibilidades de varones y mujeres de ingresar a cargos de elección popular, sino también reflexionando en torno a que estas reglas son permeadas por la cultura política que establece roles femeninos y masculinos. Esto podría generar estructuras de posibilidad diferentes de acuerdo al sexo, limitando el derecho a participar y subordinando a un determinado grupo.

Se establece que, a pesar de la inclusión de acciones afirmativas tendientes a igualar las realidades de varones y mujeres en política, ésta última sigue siendo un ámbito regido por una hegemonía masculina (Johnson 2014). Esto indica que las lógicas y las dinámicas de poder partidarias y políticas no se han modificado en función de los nuevos patrones de género.

Lo cual nos lleva a la necesidad de que, a la incorporación de medidas específicas que implementen un trato diferencial para las mujeres dentro del derecho electoral, sea acompañada de un estudio sobre la cultura política y los estereotipos, en la sociedad en general y en los partidos políticos en particular.

Dentro del IF será necesario recuperar las discusiones en torno al concepto de cultura política. Huntington y Harrison establecen la cultura en términos subjetivos, entendiendo que en ello se encuentran "los valores, las actitudes, la creencias, orientaciones y suposiciones subyacentes que prevalecen entre las personas que conforman una sociedad"(Huntington y Harrison 2001: 17). Y en esta línea las instituciones reproducen dichos valores y orientaciones.

Ahora bien, es necesario centrarse en aquellas concepciones, normas y valores enfocados hacia el ámbito específicamente político. Para Almond y Verba (1970) la cultura política son las orientaciones políticas, aquellas posturas relativas 
al sistema político y a sus elementos constitutivos, como así también al rol de cada individuo dentro de dicho sistema.

Según Heras Gómez (2002) la sociedad es el conjunto de las relaciones invisibles que los individuos establecen entre sí. Estas relaciones forman estructuras e instituciones, las cuales dan significado a la acción social, y es aquí donde entienden que se localiza la cultura política. En esta línea, Lechner(1997) propondrá que es necesario indagar los sistemas de valores, las representaciones simbólicas y los imaginarios colectivos.

El IF mostró una manera de pensar las instituciones y en ella la cultura de un sistema político definido, a partir de observar cómo eran atravesadas por el género. La pregunta que queda responder es cómo esto se tradujo en el estudio de los sistemas electorales y el género dentro de la ciencia política argentina.

\section{Sobre estudios de género y sistemas electorales en Argentina}

Para explicar el comienzo de los estudios de género y sistemas electorales y la influencia institucionalista, es necesario remontarnos a trabajos de corte historiográficos. Aquí podemos observar bibliografía referida al desarrollo de las mujeres dentro de los espacios públicos y a la concreción de ciertos derechos ejercicio del voto, participación sindical o partidaria, ejercicio del derecho a ser elegida-. Estos trabajos abordan la cuestión desde una visión histórica estableciendo de manera descriptiva los procesos y las acciones que generaron la igualación de derechos (Barrancos 2002).

En cuanto a los estudios propiamente politológicos de género, los mismos van a tener un impulso mayor a finales de siglo XX de la mano de la "Convención sobre la Eliminación de toda forma de Discriminación contra la Mujer" (CEDAW por sus siglas en inglés). Aquí la mirada estuvo puesta en la capacidad que tienen las instituciones públicas y sus recursos humanos de generar políticas públicas bajo perspectiva de género (Rigat-Pflaum 2008). Se establecieron protocolos para

Revista Zona Franca- Centro de estudios interdisciplinario sobre las mujeres (CEIM)- Maestría poder y sociedad desde la problemática de género (MG), Rosario, Argentina. ISSN, 2545-6504 http://zonafranca.unr.edu.ar/index.php/ZonaFranca| Numero 27 (2019). 
la incorporación de la mirada de género en diferentes entidades públicas, muchas veces sin la contemplación necesaria de las características particulares de los Estados y sus sociedades.

Otra vertiente de análisis es la que indaga acerca de la inclusión política de la mujer y su acceso a espacios de poder. El objetivo principal es transparentar las diferencias entre los géneros y buscar soluciones a esta problemática. Es así que se generó una importante producción académica vinculada a los porcentajes de participación de mujeres en diferentes ámbitos.

Comenzaron a desarrollarse trabajos acerca de los efectos del sistema electoral y de su capacidad para disminuir o aumentar la inclusión de mujeres en cargos electivos. La sanción de los cupos fue el puntapié inicial para abordar sus efectos. Surgen de este modo, estudios sobre cuotas de género y su desempeño a partir del sistema electoral en el que se insertan (Archenti 2000; Archenti y Tula 2008, 2014a; Marx, Borner y Caminotti 2006). Esta etapa va a estar caracterizada por su corte institucionalista, al darle una preponderancia dentro del análisis a las reglas formales por las cuales se acceden a cargos electivos.

Son estos trabajos los que establecerán como regla general que las mujeres tienen mayores posibilidades de resultar electas en sistemas proporcionales bajo circunscripciones plurinominales, con listas cerradas y donde existan legislaciones de cupo, las cuales a su vez tienen que establecer mandatos de posición en la constitución de las listas y sanciones para aquellos partidos que infringen la norma (Atela 2011).

El lugar de la ley de cupo argentina sancionada en el año 1991 abrió un campo de análisis en torno a si dicha legislación modificaba los patrones políticos establecidos en términos del género (Piatti-Crocker 2014). Esto derivó en estudios respecto de las diferentes adaptaciones que las provincias hicieron de ella, y cómo ello afectó la participación de las mujeres (Fernández y Ruiz 2014; Martin 2014).

Revista Zona Franca- Centro de estudios interdisciplinario sobre las mujeres (CEIM)- Maestría poder y sociedad desde la problemática de género (MG), Rosario, Argentina. ISSN, 2545-6504 http://zonafranca.unr.edu.ar/index.php/ZonaFranca| Numero 27 (2019). 
En esa línea surgió también el cuestionamiento al federalismo electoral argentino (Caminotti y Freidenberg 2016). El análisis de las cuotas de género subnacionales plantea la libertad que el federalismo electoral permite a la hora de la incorporación de acciones afirmativas, las cuales en algunas provincias suele distar mucho de la legislación nacional (Caminotti 2014).

La aparición mediática y política de la paridad electoral como respuesta ante lo que los cupos no lograron conseguir, promovió el desarrollo del estudio de las legislaciones sobre la paridad en Argentina (Albaine 2015; Archenti y Albaine 2018; Archenti y Tula 2014b). Nuevamente el análisis se hace sobre los porcentajes de mujeres que, luego de implementada la paridad, acceden a cargos legislativos. La novedad será que se incorpora al debate la cuestión de la violencia que este tipo de legislaciones transparental.

El recorrido anterior nos señala cómo el interés principal estuvo puesto en demostrar cuantitativamente que varones y mujeres no eran iguales a la hora de observar la conformación de los cuerpos legislativos ó de los equipos ejecutivos de gobierno. Se demostró que la incorporación de mujeres generaba diferentes aportes a la hora de, por ejemplo, el tratamiento de determinados temas dentro de los Congresos (Marx, Borner y Caminotti 2008).

Esto será criticado por Archenti (2000), quien considera que no todas las mujeres tienen que tener las mismas perspectivas o intereses, como tampoco sucede con los varones, en este sentido señala la heterogeneidad de los grupos y cómo ello impacta en las temáticas trabajadas por los legisladores.

Finalmente encontramos las discusiones que se dan en torno al lugar de los partidos políticos en esta búsqueda de igualdad, pensándolos como instituciones a las cuales las diferencias de género las atraviesan. La importancia de ellos radica en que son el primer escalón que las mujeres deben superar para pensar sus

\footnotetext{
' Los casos que se utilizan para sostener y analizar esta cuestión serán los de México y Bolivia ya que cuentan con legislaciones de paridad previas a las argentinas. Estudios sobre estas cuestiones pueden encontrarse en Albaine $(2016,2018)$ y en Freidenberg y Del Valle Pérez (2018).
}

Revista Zona Franca- Centro de estudios interdisciplinario sobre las mujeres (CEIM)- Maestría poder y sociedad desde la problemática de género (MG), Rosario, Argentina. ISSN, 2545-6504 http://zonafranca.unr.edu.ar/index.php/ZonaFranca| Numero 27 (2019). Página 205 
carreras políticas. Se comienza a indagar sobre la selección de candidatos y el lugar de las élites partidarias en la inclusión de mujeres (Caminotti, Rotman y Varetto 2014).

Se establecieron los sesgos que se hallaban dentro del proceso de selección de candidatos y que dificultaban el desempeño femenino (Johnson 2014). Estos estudios entendieron a los partidos políticos como "hacedores de candidaturas"; Archenti y Tula (2009) señalan que los partidos y alianzas electorales muestran un comportamiento "burocrático y minimalista" al aplicar las leyes de cuotas de género, consolidándose así una configuración de género en el armado de las listas como varón-varón-mujer.

Estas investigaciones que abordan la problemática de la selección de candidatos y las trayectorias políticas en clave de género discuten la necesidad de rever los ámbitos en los que el colectivo femenino intenta participar y las dificultades que tiene al hacerlo.

En lo antes dicho, se observa la carencia de estudios respecto de grupos de identidades disidentes. ¿Qué sucede con los grupos LGBTI? Esta es una línea de análisis que todavía la ciencia política en Argentina no ha logrado vincular en el área de sistemas electorales y participación política. El análisis de estos grupos se ha realizado desde una mirada sociológica sin pensar en su rol dentro de los canales políticos tradicionales de participación (Figari 2017; Maffia s/f; Simonetto 2014). El análisis de género dentro del estudio de los sistemas electorales y de la participación política se ha dado a partir de planteos binarios y heteronormados.

A su vez, el análisis de las masculinidades y su importancia en la manera de incorporarse en política por parte de varones, tampoco ha sido analizado en profundidad. La perspectiva de género ha estado direccionada a transparentar el lugar de la mujer dentro de los espacios de poder.

\section{Las alternativas latinoamericanas}

Revista Zona Franca- Centro de estudios interdisciplinario sobre las mujeres (CEIM)- Maestría poder y sociedad desde la problemática de género (MG), Rosario, Argentina. ISSN, 2545-6504 http://zonafranca.unr.edu.ar/index.php/ZonaFranca| Numero 27 (2019). 
En función de lo anterior se observa una preponderancia de la mirada institucionalista en la manera de estudiar la participación política de mujeres en Argentina. Principalmente se encuentra el análisis de normas formales, como ser leyes de cupo, reglamentaciones partidarias, proyectos legislativos; como así también composiciones de listas o de cuerpos legislativos. Por otra parte, se observa un escaso desarrollo del análisis de la cultura política argentina y de los sesgos de género que en ella pueden aparecer.

Se requiere, entonces, recuperar en este debate, las corrientes de análisis feministas latinoamericanas, ya que posibilitan el reconocimiento y evaluación de los diferentes tipos de opresión en nuestra región, bajo una matriz decolonial (Catalán Marshall 2017).

Los estudios latinoamericanos surgen como crítica a la mirada anglosajona, no obstante, se sostienen sobre teóricas como Fraser o Pateman. Estas ideas darán lugar a lo que Femenías (2012) determina como "Pensamiento Utópico Feminista", donde se busca transformar la sociedad, modificando las condiciones socio-simbólicas vigentes a partir de trastocar los roles tradicionales de las mujeres y los varones.

Estos estudios permiten repensar discursos que se sostienen en la ciencia política argentina y que han sido aprendidos y colonizados en función de ideas y conceptos del primer mundo, basados en realidades ajenas.

Retomar a estudiosas dentro de las disciplinas sociales ayuda a comprender el sentido del feminismo en nuestro contexto. Ejemplo de ello es lo que realiza Levín (2014) al utilizar los conceptos de Fraser para explicar las nociones de justicia/injusticia en América Latina".

\footnotetext{
"Levín entiende por Justicia la paridad de participación, requiriendo de acuerdos sociales que permitan participar a los individuos en calidad de pares en la sociedad. El punto crítico es la brecha existente entre la retórica de la igualdad y de la justicia de género,por un lado, y la realidad de las desigualdades y las injusticias en políticas de género en Argentina, por otro.
}

Revista Zona Franca- Centro de estudios interdisciplinario sobre las mujeres (CEIM)- Maestría poder y sociedad desde la problemática de género (MG), Rosario, Argentina. ISSN, 2545-6504 http://zonafranca.unr.edu.ar/index.php/ZonaFranca| Numero 27 (2019). 
Por su parte, Serret (2012) establece que la sociedad democrática es un orden institucional, formal, con un cuerpo de valores y principios axiológicos, en donde la igualdad y la libertad son entendidas como universales. Para esta autora, la crítica feminista busca deconstruir este relato, para así dar cuenta de cómo dicha sociedad se sustenta en la subordinación de mujeres. Este punto es difícil encontrarlo en los trabajos de corte politológicos.

A su vez, la corriente latinoamericana proporciona un encuadre metodológico para incorporar la mirada respecto de los grupos disidentes. Constantemente se pone en discusión el reduccionismo de determinados análisis respecto de la utilización del concepto de género aplicado a la variable mujer, incluso pensando mujer en términos patriarcales (Lagarde 1997).

En esta línea se critica la homogeneidad con que se usan los conceptos, lo cual si luego pensamos dentro de la disciplina podemos observar cómo se espera que las mujeres trabajen determinados temas o respondan de la misma manera a determinadas demandas.

El sumar esta perspectiva al análisis de los sistemas electorales y la participación política, implica considerar que la cultura política y las instituciones en América Latina tiene que ser estudiada desde la diversidad de las sociedades latinoamericanas. Lo que supone dar una mayor importancia en el estudio a la historicidad, a la diversidad de discursos y a las subjetividades de los actores (Lechner 1996; Millán La Rivera 2008).

En función de lo antes mencionado, podríamos establecer que, para pensar la incorporación de mujeres y varones en espacios de poder, debemos primero observar las prácticas políticas de los actores, y en ello la cultura política argentina. Lo cual permitirá observar sesgos de género que se sostienen en el ámbito político y que son estructurantes y particulares de una determinada sociedad. Estos sesgos demuestran las relaciones de poder que se reproducen a lo largo de la sociedad.

Revista Zona Franca- Centro de estudios interdisciplinario sobre las mujeres (CEIM)- Maestría poder y sociedad desde la problemática de género (MG), Rosario, Argentina. ISSN, 2545-6504 http://zonafranca.unr.edu.ar/index.php/ZonaFranca| Numero 27 (2019). 
Es así que puede observarse una mirada social sobre la mujer en donde se entiende la existencia de ciertas características atribuibles a las mujeres (que el varón no tiene) y que ante su inclusión permitirán un cambio positivo en la práctica política (Del Valle 2008). Esto generaría estereotipos sobre cómo la mujer debe participar en política, sosteniendo así la reproducción de un Estado patriarcal y generando un "vacío de conciencia política de género en las mujeres, aunado al machismo [...]" (Lagarde 1999:3).

En esta línea Astelarra explica que ya ha sido demostrada la existencia de sesgos que reflejan prejuicios androcéntricos: "El principal de ellos es la consideración de la conducta masculina como parámetro de la "normalidad" política. [...] los comportamientos femeninos que no fueran iguales son considerados como ejemplos de desviación" (Astelarra 1987: 149).

Para esta autora, la cultura política también se expresa a partir de las actividades políticas (las cuales deben ser miradas desde la división sexual del trabajo, ya que esta reproduce el sistema de género vigente). De esta manera, se determinan espacios sociales femeninos y masculinos, y así también formas de relacionarse entre varones y mujeres, lo cual tiene su traducción en determinadas prácticas políticas.

Por lo que estos sesgos, la cultura política, las prácticas y la identificación sobre cómo debe ser la mujer y el varón en política, podrán observarse en función no sólo de la composición de las listas y de la resistencia pública a legislaciones de cuotas, sino también a través de la reconstrucción de las carreras y de la observación sobre la participación en diferentes espacios políticos. Visibilizar los ámbitos de participación política permitirá mostrar el lugar que ocupa cada individuo dentro de su espacio y determinar verdaderamente cuáles son las nociones de género vigentes en dicho sistema.

\section{Conclusiones}

Revista Zona Franca- Centro de estudios interdisciplinario sobre las mujeres (CEIM)- Maestría poder y sociedad desde la problemática de género (MG), Rosario, Argentina. ISSN, 2545-6504 http://zonafranca.unr.edu.ar/index.php/ZonaFrancal Numero 27 (2019). 
Con el presente trabajo se intentó reconstruir las discusiones actuales que vinculan los sistemas electorales y la perspectiva de género, dentro de la ciencia política argentina. A partir de la impronta institucionalista que ha tomado la disciplina se observa un desarrollo importante en términos de análisis de instituciones formales (constituciones, leyes de cuotas o paritarias, proyectos de leyes) como así también el estudio de las composiciones de listas y de los Congresos en términos binarios.

Con esto último se observa una laguna dentro de la ciencia política, la cual no da cuenta de las discusiones respecto de las sexualidades disidentes. ¿Cómo se representan políticamente estos grupos? ¿Bajo qué sistemas electorales aumenta su participación? ¿Cuál es el lugar de las masculinidades? Éstas son algunas preguntas que todavía no tienen respuesta dentro de la disciplina.

A pesar de ello, mucho se ha escrito sobre cómo los sistemas electorales argentinos afectan la manera de incorporarse en política que tienen las mujeres. Pero poco se ha abordado respecto del modo en que lo informal define dónde se participa y por qué se lo hace de esa forma.

Este trabajo intentó pensar en las teorías que desde la corriente feminista latinoamericana se gestan en torno a conceptos como cultura política, prácticas políticas y sesgos de género. Éstas permiten ampliar el marco de referencia sobre el que los cientistas políticos argentinos se posicionan para pensar participación política y la injerencia en ella del sistema electoral.

En este trabajo se considera que estas teorías, construidas desde la periferia, brindan directrices para pensar las características particulares del contexto argentino y latinoamericano, ya que las mismas no pueden ser analizadas desde teorías construidas en el mundo anglosajón.

Es así que se considera que estos conceptos permitirán observar las cuestiones que permean a la norma, las cuestiones que no permiten que los cupos 
se den, las creencias y normatividades sociales que se reproducen en el ámbito político.

Desde la academia se sostiene el valor democrático, como un orden institucional y formal, donde la igualdad y la libertad son entendidas como universales. Para ello será necesario retomar el concepto de Democracia Genérica construido por Lagarde (1997), éste plantea construir concepciones de mundo (que se traduzcan al ámbito político) que no estén basadas en la opresión de género.

Los cupos o legislaciones electorales paritarias deberán estar acompañadas por un análisis sincero de la realidad política y partidaria, intentando transparentar la cultura política argentina; para que, de esa manera se lleven adelante legislaciones posibles en términos de equiparación de derechos entre varones y mujeres.

A su vez será necesario vincular la cuestión del federalismo electoral argentino con las culturas subnacionales. No es lo mismo ser mujer o varón en Salta que en Buenos Aires, y los cupos legislativos o electorales también reproducen esas diferencias. Repensar estas situaciones permitirá acercarse al concepto de Democracia Genérica.

Es por ello que en el presente trabajo se intentó transparentar los vínculos entre institucionalismo y ciencia política argentina, analizando cómo el primero influyó en la manera de generar conocimiento en torno a sistemas electorales y participación política.

Se considera que el rol desde la ciencia política con perspectiva feminista debe ser deconstruir el relato tradicional, para así dar cuenta de cómo la sociedad política también se sustenta en la subordinación de determinados grupos -como ser la mujer- (Serret 2012).

Revista Zona Franca- Centro de estudios interdisciplinario sobre las mujeres (CEIM)- Maestría poder y sociedad desde la problemática de género (MG), Rosario, Argentina. ISSN, 2545-6504 http://zonafranca.unr.edu.ar/index.php/ZonaFranca| Numero 27 (2019). 
Los relatos desde Latinoamérica pueden ayudar en dicha deconstrucción, planteando las particularidades de los territorios y cómo ello afecta la manera de generar legislaciones. Estas cuestiones no suelen estar planteadas dentro de las investigaciones sobre sistemas electorales y este trabajo fue un intento de repensarnos en ese sentido.

\section{Bibliografía}

ALMOND, Gabriel y VERBA, Sidney (1970) La cultura Cívica. Estudio sobre la participación política democrática en cinco naciones". España: Fundación FOESSA.

ALBAINE, Laura (2015) "Obstáculos y desafíos de la paridad política de género" en Revista Iconos; Quito; p. 145 - 162.

ALBAINE, Laura (2016) "Paridad de género y violencia política en Bolivia, Costa Rica y Ecuador. Un análisis testimonial", en Revista de ciencias políticas de la Universidad Nacional de Colombia; Bogotá; vol. 11 p. 335 - 363.

ALBAINE, Laura "Estrategias legales contra la violencia política de género. Las oportunidades de acción" en Revista de Estudios de Género, La Ventana; Guadalajara; p. 264 - 293.

ARCHENTI, Nélida (2000) "Representación, Ley de Cuotas y Sistemas Electorales"; en Revista PostData, № 6, Buenos Aires.

ARCHENTI, Nélida y ALBAINE, Laura (2018) "O Feminismo na política. Paridade e violência política de gênero na América Latina" en Cadernos Adenauer XIX, №1; Rio de Janeiro; p. 9-24.

ARCHENTI, Nélida y TULA, María Inés (2008) Mujeres y Política en América Latina, Sistemas Electorales y cuotas de género, Buenos Aires: Editorial Heliasta.

ARCHENTI, Nélida y TULA, María Inés (2009) "Representación política, sistemas electorales y género. Análisis de las listas partidarias en cinco distritos subnacionales, Argentina 2007", en IX Congreso Nacional de Ciencia Política. Organizado por la Sociedad Argentina de Análisis Político y la Universidad Nacional del Litoral. Santa Fe.

Revista Zona Franca- Centro de estudios interdisciplinario sobre las mujeres (CEIM)- Maestría poder y sociedad desde la problemática de género (MG), Rosario, Argentina. ISSN, 2545-6504 http://zonafranca.unr.edu.ar/index.php/ZonaFranca| Numero 27 (2019). 
ARCHENTI, Nélida y TULA, María Inés (2014a) La representación política imperfecta. Logros y desafíos de las mujeres políticas. Editorial EUDEBA. Buenos Aires.

ARCHENTI, Nélida y TULA, María Inés (2014b)“Cambios normativos y equidad de género. De las cuotas a la paridad en América Latina: los casos de Bolivia y Ecuador." En América Latina Hoy. Salamanca; vol. 66 p. 47 - 68.

ASTELARRA, Judith (1987) "La cultura política de las mujeres", en LECHNER, Norbert (comp), Cultura política y democratización, Santiago de Chile: CLACSO.

ATELA, Vicente (2011) "Los sistemas electorales en el derecho público provincial argentino" en ANALES, $N^{\circ}$ 41. pp. 213-228.

BARRANCOS, Dora (2002) Inclusión/Exclusión. Historia con Mujeres. Fondo de Cultura Económica. Buenos Aires.

BUCKLEY, Fiona; MARIANI, Mack y WHITE, Timothy (2014) "Will legislative gender quotas increase female representation in Ireland? A feminist institutionalism analysis", en Representation, Londres, 50:4.

CAMINOTTI, Mariana (2014) "No todos los caminos van a Roma. Leyes de cuota, federalismo y diversidad institucional en Argentina" en Modus Vivendi. Estado federal y política multinivel en Argentina. Buenos Aires; p. 255 - 280.

CAMINOTTI, Mariana y FREIDENBERG ， Flavia (2016) "Federalismo electoral, fortaleza de las cuotas de género y representación política de las mujeres en Argentina y México" en Revista Mexicana de Ciencias Políticas y Sociales; Lugar: Ciudad de México; p. 121 - 144.

CAMINOTTI, Mariana; ROTMAN, Santiago y VARETTO, Carlos (2014) "Desigualdades persistentes: una mirada a las carreras políticas subnacionales antes y después de las cuotas de género (Argentina, 1983-2011)" en ARCHENTI, Nélida y TULA, María Inés (coord.) La representación política imperfecta. Logros y desafíos de las mujeres políticas, Buenos Aires: Editorial EUDEBA.

CATALÁN MARSHALL, Mario Alejandro (2017) "Feminismos y Estudios de Género en el Sur del mundo. Reflexiones críticas en torno a la Colonialidad en la

Revista Zona Franca- Centro de estudios interdisciplinario sobre las mujeres (CEIM)- Maestría poder y sociedad desde la problemática de género (MG), Rosario, Argentina. ISSN, 2545-6504 http://zonafranca.unr.edu.ar/index.php/ZonaFranca| Numero 27 (2019). 
teoría y en la práctica feminista latinoamericana", en RevIISE, San Juan, Vol 9 N $^{\circ}$ 9.

DAHLERUP, Drude (2002) "El uso de cuotas para incrementar la representación política de la mujer", en KARAM, Azza, Mujeres en el Parlamento. Más allá de los números, Suecia: IDEA Internacional.

DUVERGER, Maurice (1955) The political role of women. París: UNESCO.

DEL VALLE, Teresa (2008) "La cultura del poder desde y hacia las mujeres", en BULLEN, Margaret Louise y DIEZ MINTEGUI, María Carmen (coord.) Retos Teóricos y Nuevas Prácticas, España: Universidad del País Vasco/Euskal Herriko Unibertsitatea.

FERNÁNDEZ, Elías y Ruiz, Paola (2014) “ Las mujeres en la política entrerriana. Representación de género en la legislatura provincial (1983-2011)" en ARCHENTI, Nélida y TULA, María Inés (coord.) La representación política imperfecta. Logros y desafíos de las mujeres políticas, Buenos Aires: Editorial EUDEBA.

FEMENÍAS, Luisa (2012) "Feminismo y utopía: una labor constante", en Equipo Latinoamericano de Justicia y Género, Autonomía y feminismo siglo XXI, escritos en homenaje a Haydée Birgin, Buenos Aires: Editorial Biblos.

FIGARI, Carlos Eduardo (2017) "Consideraciones sobre el movimiento LGBT en Argentina", en Boletín Onteaiken N²4. Argentina.

FREIDENBERG, Flavia y DEL VALLE PÉREZ, Gabriela (edit) (2018) Cuando hacer política te cuesta la vida. Estrategias contra la violencia política hacia las mujeres en América Latina; Universidad Nacional Autónoma de México, Instituto de Investigaciones Jurídicas y Tribunal Electoral de la Ciudad de México; México.

HERAS GÓMEZ, Leticia (2002) "Cultura política: el estado del arte contemporáneo", en Convergencia. Revista de Ciencias Sociales, Toluca, Vol. 9 N³0.

HUNTINGTON, Samuel y HARRISON, Lawrence (2001) La cultura es lo que importa. Cómo los valores dan forma al progreso humano, Buenos Aires: Editorial Planeta.

Revista Zona Franca- Centro de estudios interdisciplinario sobre las mujeres (CEIM)- Maestría poder y sociedad desde la problemática de género (MG), Rosario, Argentina. ISSN, 2545-6504 http://zonafranca.unr.edu.ar/index.php/ZonaFranca| Numero 27 (2019). 
JOHNSON, Niki (2014) "Las barreras que la cuota no superó: selección de candidatos y desigualdades de género en América Latina", en ARCHENTI, Nélida y TULA, María Inés (coord.) La representación política imperfecta. Logros y desafíos de las mujeres políticas, Buenos Aires: Editorial EUDEBA.

KROOK, Mona Lena (2009) Quotas for Women in Politics: Gender and Candidates Selection Reform Worldwide. Oxford University Press. New York.

KROOK, Mona Lena y MACKAY, Fiona (2011) "Introduction: Gender, Politics and institutions" en KROOK, Mona Lena y MACKAY, Fiona (eds), Gender, Politics and Institutions. Towards a Feminist Institutionalism, Gran Bretaña: Palgrave Macmillan.

LAGARDE, Marcela (1997) Género y Feminismo. Desarrollo Humano y Democracia. España: Horas y horas la editorial.

LAGARDE, Marcela (1999) "Claves identitarias de las latinoamericanas en el umbral del milenio", en PORTUGAL, Ana María y TORRES, Carmen (Eds), El siglo de las mujeres, Santiago de Chile: Isis Internacional Ediciones de las Mujeres. Santiago de Chile.

LECHNER, Norbert (1997), "El malestar con la política y la reconstrucción de los mapas políticos", en WINOCUR, Rosalía (coord.), Culturas políticas a fin de siglo, México D.F.: Juan Pablos Editor-FLACSO.

LEVIN, Silvia (2014) "Capitulo 2: Dilemas de justicia política en Argentina: ¿Representación, libertad?", en CARBONERO GAMUNDÍ, María Antonia y LEVIN, Silvia (coord.), Injusticias de género en un mundo globalizado. Conversaciones con la teoría de Nancy Fraser. Buenos Aires: Homo Sapiens Ediciones.

LLANOS, Beatriz y SAMPLE, Kristen (2008) 30 Años de democracia: ¿En la cresta de la ola? Participación política de la mujer en América Latina, Perú: IDEA Internacional.

MACKAY, Fiona, KENNY, Meryl y CHAPELL, Louise (2010) "New institutionalism through a Gender lens: Towards a feminist institutionalism?" en International Political Science Review, Londres: Sage Publications, 31 (5).

Revista Zona Franca- Centro de estudios interdisciplinario sobre las mujeres (CEIM)- Maestría poder y sociedad desde la problemática de género (MG), Rosario, Argentina. ISSN, 2545-6504 http://zonafranca.unr.edu.ar/index.php/ZonaFrancal Numero 27 (2019). 
MAFFIA, Diana (s/f) "Paridad en la participación política de las mujeres y otros grupos discriminados por su género", en https://www.argentina.gob.ar/sites/default/files/diana maffia.pdf

MARCH, James G. y OLSEN, Johan P. (1993) "El Nuevo institucionalismo: factores organizativos de la vida política" en Zona Abierta 63/64. España: Editorial Pablo Iglesias.

MARTíN, María Elena (2014) "Diez años sin cupo, diez años con cupo: ¿Qué cambió con la cuota de género? Un análisis de la provincia de Misiones 19832003" en ARCHENTI, Nélida y TULA, María Inés (coord.) La representación política imperfecta. Logros y desafíos de las mujeres políticas, Buenos Aires: Editorial EUDEBA.

MARX, Jutta, BORNE, Jutta y CAMINOTTI, Mariana (2006) "Cuotas de género y acceso femenino al Parlamento. Los casos de Argentina y Brasil en perspectiva comparada" en Política (Revista de Ciencia Política de la Universidad de Chile). Vol. 46. Santiago de Chile.

MILLÁN LA RIVERA, Cecilia (2008) "Cultura Política: Acercamiento conceptual desde América Latina", en Perspectivas de la comunicación, Temuco: Universidad de la Frontera, Vol. 1, № 1.

NORRIS, Pippa (1985) “Women's legislative participation in Western Europe”, West European Politics N8. Pp. 90-101.

PATEMAN, Carole (1996) Críticas feministas a la dicotomía público-privado, Barcelona: Paidós.

PETERS, Guy (1999) Institutional Theory in Political Science: The New Institutionalism. Londres: Editorial Continnum.

PHILLIPS, Anne (1996) "¿Deben las feministas abandonar la democracia liberal?" en CASTELLS, Carme (comp), Perspectivas feministas en teoría política, España: Ediciones Paidós lbérica.

PIATTI-CROCKER, Adriana (2014) "Dos décadas de cuotas de género en Argentina: difusión de políticas y sus efectos" en ARCHENTI, Nélida y TULA, 
María Inés (coord.) La representación política imperfecta. Logros y desafíos de las mujeres políticas, Buenos Aires: Editorial EUDEBA.

RIGAT-PFLUAM, Maria (2008) "Gender mainstreaming: un enfoque para la igualdad de género" en Revista Nueva Sociedad. N 218. Acceso web: www.nuso.org

SARTORI, Giovanni (2007) ¿Qué es la Democracia?.España: Editorial Taurus.

SARTORI, Giovanni (2009) La democracia en 30 lecciones. España: Editorial Taurus.

SERRET, Estela (coord.) (2012) Democracia y Ciudadanía: Perspectivas críticas feministas, México: Editorial Fontamara.

SIMONETTO, Patricio (2014) "Los fundamentos de la revolución sexual: teoría y política del Frente de Liberación Homosexual en la Argentina (1967-1976)" en Anuario de la Escuela de Historia Virtual - Año 5 - No 6 - 2014: pp. 150-174. Argentina: Universidad Nacional de Córdoba.

Revista Zona Franca- Centro de estudios interdisciplinario sobre las mujeres (CEIM)- Maestría poder y sociedad desde la problemática de género (MG), Rosario, Argentina. ISSN, 2545-6504 http://zonafranca.unr.edu.ar/index.php/ZonaFrancal Numero 27 (2019). 